Research Article

\title{
Solving Power Economic Dispatch Problem with a Novel Quantum-Behaved Particle Swarm Optimization Algorithm
}

\author{
Li Ping $\left(\mathbb{D},{ }^{1}\right.$ Jun Sun $\mathbb{D}^{2},{ }^{2}$ and Qidong Chen $\mathbb{D}^{2}$ \\ ${ }^{1}$ IoT School, Wuxi Institute of Technology, Wuxi 214121, China \\ ${ }^{2}$ IoT School, Jiangnan University, Wuxi 214122, China \\ Correspondence should be addressed to Li Ping; cqd_jnu@hotmail.com
}

Received 5 July 2020; Revised 3 November 2020; Accepted 1 December 2020; Published 11 December 2020

Academic Editor: Luis Payá

Copyright $(0) 2020$ Li Ping et al. This is an open access article distributed under the Creative Commons Attribution License, which permits unrestricted use, distribution, and reproduction in any medium, provided the original work is properly cited.

\begin{abstract}
This paper proposes the shrink Gaussian distribution quantum-behaved optimization (SG-QPSO) algorithm to solve economic dispatch (ED) problems from the power systems area. By shrinking the Gaussian probability distribution near the learning inclination point of each particle iteratively, SG-QPSO maintains a strong global search capability at the beginning and strengthen its local search capability gradually. In this way, SG-QPSO improves the weak local search ability of QPSO and meets the needs of solving the ED optimization problem at different stages. The performance of the SG-QPSO algorithm was obtained by evaluating three different power systems containing many nonlinear features such as the ramp rate limits, prohibited operating zones, and nonsmooth cost functions and compared with other existing optimization algorithms in terms of solution quality, convergence, and robustness. Experimental results show that the SG-QPSO algorithm outperforms any other evaluated optimization algorithms in solving ED problems.
\end{abstract}

\section{Introduction}

Solving economic dispatch (ED) problem is to ensure that the power production is safe, high-quality and meets the customer's electricity demand by using various technical and management measures to make the power production equipment in the best working state and reach the lowest cost of the power system. Simultaneously, the nonlinear characteristics of the generator, such as ramp rate limit, prohibited operating area, and nonsmooth cost function, should be considered. Therefore, the ED problem is a complex nonlinear problem with many constraints.

Traditionally, the ED problem can be solved by various mathematical programming methods, including lambda iterative method, the base point [1], the interior point method [2], the gradient method [3], and the dynamic programming method [4]. However, these deterministic numerical methods do not work effectively for problems with hard constraints such as nonsmooth and nonconvex cost functions, or suffer "dimensional disasters." Therefore, in order to effectively address the issues of the nonlinear characteristics of practical power systems, many swarm intelligence algorithms or evolutionary algorithms are used to solve multiconstrained optimization problems, including genetic algorithms (GA) [5], particle swarm optimization (PSO) [6], differential evolution (DE) [7], evolutionary programming (EP) [8,9], tabu search (TS) [10], neural network (NN) $[11,12]$, ant colony search algorithm (ACSA) [13], artificial immune system (AIS) [14, 15], honey bee colony algorithm [16], firefly algorithm [17], and the hybrid method [18].

Besides, some improved algorithms are proposed. For example, Kaboli proposes an artificial cooperative search (ACS) [19] optimization algorithm, which is provided by balancing exploration of the problem's search space and exploitation of better results through the use of two advanced evolutionary operators and only one control parameter. Pandey proposes an improved FWA with Chaotic Sequence Operator (IFWA-CSO) [20], in which the global search ability of FWA has been strengthened. Sun uses the improved particle swarm optimization algorithm RDPSO (Random Drift Particle Swarm Optimization) to solve the power 
optimization problem [21]. In order to improve the local search capability of PSO, KHAMSAWANG and Grag adopt a hybrid method of differential evolution or genetic algorithm to enhance the local capability $[22,23]$. Besides, Khan et al. control the diversity of particle swarm to avoid the algorithm falling into the local optimal [24-26]. Besides, other improved algorithms such as the iterated-based optimization method [27], dynamically controlled particle swarm optimization method [28] are also performed well on ED problems.

However, for these methods, the major deficiencies still are highly sensitive to the initial value of the control parameters and a large number of control parameters or some situations such as trap into local optima and premature easily occur. Therefore, it is difficult to obtain satisfactory and feasible solutions for multiconstrained, nonlinear optimization problems. The quantum-behaved particle swarm optimization (QPSO) algorithm is a variant PSO algorithm that has strong and robust global search ability but has relatively low convergence speed and local search ability.

Therefore, this paper proposes a shrink Gaussian distribution quantum-behaved optimization (SG-QPSO) algorithm to solve ED problems from the power systems area. By shrinking the Gaussian probability distribution near the learning inclination point of each particle iteratively, SGQPSO not only maintains a strong global search capability at the early search stage but also strengthens the local search capability at the later stage. In this way, the proposed SGQPSO improves the weak local search ability of QPSO and meets the needs of solving the ED optimization problem at different stages. Besides, SG-QPSO has fewer parameters than other optimization algorithms, such as genetic algorithms, differential evolution, or other one-dimensional search algorithms like the Powell algorithm, which is easier to control.

The remaining chapters of this paper are arranged as follows: Section 2 describes the proposed SG-QPSO in detail. Section 3 describes the mathematical formulation of the ED problem in detail. Section 4 shows the experimental results obtained by SG-QPSO on three power systems, compares its results with previous algorithms, and analyzes its merits and disadvantages. Section 5 summarizes this article and introduces the focus of future work.

\section{The Proposed Algorithm}

In this section, we first introduce the theoretical aspect of the canonical QPSO algorithm and then analyze its advantages and disadvantages when dealing with ED problems. Based on the analysis above, an improved QPSO algorithm called SG-QPSO is proposed, and its process of solving the ED problem is given as a flowchart.

2.1. QPSO Algorithm. The crucial issue of QPSO is how to design a reasonable potential energy field [29]. Clerc analyzed the dynamic evolution process and showed that each particle gradually converges to a point [30]. In other words, those points attract the particles swarm during the search process. Those points are named learning inclination points (LIPs) in QPSO, and its current position is calculated as follows:

$$
p_{i, t}^{j}=\varphi_{i, t}^{j} \cdot P_{i, t}^{j}+\left(1-\varphi_{i, t}^{j}\right) G_{t}^{j},
$$

where $\varphi_{i, t}^{j} \in(0,1)$ is a random variable generated by uniform distribution, $P_{i, t}^{j}$ is the value that denotes the $j$-th dimension of the current personal best position of particle $i$, and $G_{t}^{j}$ represents the value of the $j$ th dimension of the current global best position.

The updated formulation of each particle in QPSO is as follows:

$$
X_{i, t+1}^{j}=p_{i, t}^{j} \pm 0.5 \cdot L_{i, t}^{j} \cdot \ln \left(\frac{1}{u_{i, t+1}^{j}}\right),
$$

where $u_{i, t}^{j} \in(0,1)$ is a random variable generated by uniform distribution, $L_{i, t}^{j}$ is the length between the current position of each particle and the mean personal best position, and its definition is as follows:

$$
\begin{aligned}
& L_{i, t}^{j}=2 \cdot \alpha_{t} \cdot\left|C_{t}^{j}-X_{i, t}^{j}\right|, \\
& \alpha_{t}=\alpha_{0}+\left(\alpha_{1}-\alpha_{0}\right) \cdot \frac{T-t}{T} .
\end{aligned}
$$

The mean personal best position is calculated by the following:

$$
C_{t}^{j}=\sum_{1}^{M} P_{i, t}^{j} .
$$

By simulating the strong uncertainty of the superposition of states in the quantum system, QPSO makes it possible to cover the whole probability search space during the search process. Simultaneously, the algorithm uses the mean personal best position to guide the particles to gradually aggregate to LIPs. This delay strategy makes the algorithm convergence slowly and helps the algorithms enhance their global search ability. The details of the QPSO algorithm can be found in [29].

2.2. The SG-QPSO Algorithm. When the area near the global or local optima is tiny, particles in QPSO are easier to skip this area for the range of update area of each particle is large. At the same time, considering that Gaussian distribution is introduced to generate random variables sequence may weaken the global search ability, a shrink Gaussian distribution quantum-behaved particle swarm optimization (SG-QPSO). In SG-QPSO, the variance of Gaussian distribution declines linearly to shrink the area of each particle near its LIP, which enhances the local search ability gradually and maintains the global search ability of QPSO during the search process. The update formula of the SG-QPSO algorithm is as follows:

$$
\begin{cases}X_{i, t+1}^{j}=p_{i, t}^{j}+\left|C_{t}^{j}-X_{i, t}^{j}\right| \cdot \ln \left(\frac{1}{N_{i, t+1}^{j}(0, \sigma)}\right), & \text { if } k<0.5, \\ X_{i, t+1}^{j}=p_{i, t}^{j}-\left|C_{t}^{j}-X_{i, t}^{j}\right| \cdot \ln \left(\frac{1}{N_{i, t+1}^{j}(0, \sigma)}\right), & \text { otherwise, }\end{cases}
$$

where $k$ is random values generated by using the uniform probability distribution functions in the range $[0,1]$. The 
learning inclination point $p$ and mean personal best position $C$ is calculated by equations (1) and (4). Note that the number of particles in a particle swarm is $M$ :

$$
\sigma_{t}=\sigma_{0}+\left(\sigma_{1}-\sigma_{0}\right) \cdot \frac{T-t}{T}
$$

In equation (6), $\sigma$ denotes the variance of Gaussian distribution, declining linearly from the initial value $\sigma_{1}$ and the end value $\sigma_{0}$ in the search process. $T$ is the maximum number of fitness evaluations and $t$ represents the current iteration step. The Pseudocode of the SG-QPSO algorithm is shown in Algorithm 1.

\section{Solving ED Problem with SG-QPSO}

3.1. Mathematical Model of Power System Economic Dispatch. The ED problem can be reduced to an optimization problem. Its goal is to determine the power output level of the online generator and further minimize the total fuel cost of all generators within a period while satisfying various nonlinear constraints.

3.1.1. Objective Function. The objective function of ED problem can be defined as follows:

$$
\operatorname{minimize} F_{\text {cost }}=\sum_{j=1}^{N_{g}} F_{j}\left(P_{j}\right),
$$

where $F_{j}\left(P_{j}\right)$ is the cost function of $j$ th generator set, $P_{j}$ Is the actual output of the $j$ th generator set, and $N_{g}$ is the total number of generators in the power system.

The cost function of each generator set is related to the actual power put into the system and is usually modeled with a smooth quadratic function:

$$
F_{j}\left(P_{j}\right)=a_{j}+b_{j} P_{j}+c_{j} P_{j}^{2}
$$

$a_{j}, b_{j}$, and $c_{j}$ is the cost correlation coefficient of the $j$ th generator set.

3.1.2. Constrains of ED Problem. In this work, we consider the following constraints of the ED problem:

(a) Power balance constraints

The power balance constraints are expressed as follows:

$$
\sum_{j=1}^{N_{g}} P_{j}=P_{D}+P_{L}
$$

The total power generation of the system is equal to the load demand of the system plus transmission loss. In other words, the total power generation $\sum_{j=1}^{N_{g}} P_{j}$ should be equal to the total power demand $P_{D}$ plus transmission network loss $P_{L}$ while minimizing total power generation costs. $P_{L}$ is usually approximated by the Krone loss formula, which represents the relationship between the transmission loss and the output level of the system generator set:

$$
P_{L}=\sum_{j=1}^{N_{g}} \sum_{k=1}^{N_{g}} P_{j} B_{j k} P_{k}+\sum_{j=1}^{N_{g}} P_{j} B_{j 0}+B_{00}
$$

where $1 \leq j, k \leq N_{g}$ the number of generators in the generator set, $B_{j 0}, B_{j k}, B_{00}$ is called loss coefficient. $B_{j k}$ is a $N_{g} \times N_{g}$ matrix.

(b) Inequality constraints

$$
P_{j}^{\min }<P_{j}<P_{j}^{\max }, \quad\left(j=1,2, \ldots, N_{g}\right) .
$$

According to the design requirements of the generator, the amount of power generated by each unit must vary between its minimum $P_{j}^{\min }$ and maximum $P_{j}^{\max }$ production limits.

(c) Ramp rate limitation

During the actual operation of the generator set, the operating range of all online units is limited by their ramp rate limitation. According to [5], the inequality constraint due to the slope limitation is as follows:

(i) If the amount of power generation increases

$$
P_{j}-P_{j}^{0} \leq U R_{j}
$$

(ii) If the amount of power generation decreases

$$
P_{j}^{0}-P_{j} \leq D R_{j}
$$

(d) Prohibited operating areas

Since the steam valve operates in the bearing (i.e., vibration), the system contains some prohibited operating zones. In the actual power system, the load demand of the power system must avoid prohibited zones. Therefore, if the constraints in (11) are considered, the feasible operating area of the $j$ th generator set can be described in the following way:

$$
\begin{gathered}
P_{j}^{\min } \leq P_{j} \leq P_{j, 1}^{l}, \\
P_{j, k-1}^{u} \leq P_{j} \leq P_{j, k}^{l}, \quad k=2,3, \ldots, n_{j}, \\
P_{j, n_{j}}^{u} \leq P_{j} \leq P_{j}^{\max },
\end{gathered}
$$

where $P_{j, k}^{l}$ and $P_{j, k}^{u}$ is The upper and lower boundaries of the $k$ th prohibited zone of the $j$ th generator set, $n_{j}$ is the number of prohibited zones of the $j$ th generator set.

3.1.3. The Mathematical Formulation of ED Problem. Combining the equations (11)-(13), we obtained the following:

$$
\operatorname{Max}\left(P_{j}^{\min }, P_{j}-D R_{j}\right) \leq P_{j} \leq \min \left(P_{j}^{\max }, P_{j}^{0}+U R_{j}\right) .
$$


Therefore, considering the feasible operation zones, we can express the ED problem as the following constrained optimization problem:

$$
\operatorname{minimize} F_{\text {cost }}=\sum_{j=1}^{N} F_{j}\left(P_{j}\right)
$$

subject to

$$
\begin{aligned}
& \sum_{j=1}^{N_{g}} P_{j}=P_{D}+P_{L} \\
& \max \left(P_{j}^{\min }, P_{j}-D R_{j}\right) \leq P_{j} \leq P_{j, 1}^{l} \\
& P_{j, k-1}^{u} \leq P_{j} \leq P_{j, k}^{l}, k=2,3, \ldots, n_{j} \\
& P_{j, n_{j}}^{u} \leq P_{j} \leq \min \left(P_{j}^{\max }, P_{j}^{0}+U R_{j}\right) .
\end{aligned}
$$

3.2. Solving ED Problem Using SG-QPSO. Before applying the SG-QPSO algorithm to the ED problem, make the following provisions:

Each component in a single particle represents a generation unit, so each particle represents a candidate solution for a given ED problem. The current position of the th particle $P_{g, i}$ with $N_{g}$ generation units can be given by the following:

$$
P_{g, i}=\left[P_{i, 1}, P_{i, 1}, P_{i, 2}, \ldots, P_{i, N_{g}}\right], \quad i=1,2, \ldots, M,
$$

where $M$ is the population size, which is the index to generate $j$ th unit, and $P$ is the output power of the $i$ th generating unit in the $j$ th particle.

3.2.1. Objective Function and Constraint Handling. The equality constraints in the formula can be handled by adding penalty terms. The objective function becomes as follows:

$$
\operatorname{minimize} F=\sum_{j=1}^{N} F_{j}\left(P_{j}\right)+K_{n}\left|\sum_{j=1}^{N} P_{j}-P_{D}-P_{L}\right|,
$$

where $K_{n}$ is called the penalty coefficient and is a positive real number, which increases with the number of iterations. The penalty term in equation (18) is the equality constraint in equation (9). When the ED problem is restricted, it is solved by a population-based search method (such as SGQPSO). If the equality constraint is violated, the value of the penalty term is nonzero.

On the one hand, when the candidate solution violates the equation-constrained candidate solution, equation (18) gives a larger objective function value so that the candidate solution has a greater probability of being discarded. On the other hand, when the equality constraint is not violated, the penalty term is zero. No matter how large the penalty coefficient is, the final penalty term value is zero. Therefore, the final objective function value is obtained by adding the value of the penalty term to the given objective function value so as to control each candidate solution in the population to approach the feasible solution area.

\section{Experiments}

4.1. The Summary of Three Power Systems. Three real power systems are used to verify the effectiveness of SG-QPSO, with considering the ramp rate limit and the prohibited zones. Other optimization methods are also tested on these three systems for comprehensive performance comparison, including binary-coded GA [5], PSO with inertial weights [6], DE [7], Ant Colony Search Algorithm (ACSA) [13], artificial immune system (AIS) [14], bee colony optimization (BCO) [16], firefly algorithm (FA) [17], standard PSO (SPSO) with shrinkage and inertial weights [31], chaotic PSO (CPSO) [32], antipredatory PSO (APSO) [33], mixed gradient descent PSO (HGPSO) [31], mixed PSO with mutation (HPSOM) [31], QPSO [29], and GQPSO [34]; the Hopfield neural network (NN) was also tested. Note that, for each system, all test methods use the same objective function.

System 1: the system consists of 6 thermal units, 26 bus bars, and 46 transmission lines. The load demand is $1263 \mathrm{MW}$. The characteristics of the 6 thermal units are given in Tables 1 and 2. In the normal operation of the system, the loss factor with a basic capacity of $100 \mathrm{MVA}$ is shown in Table 3. It is a small system and is the easiest problem among three test systems, and the dimension of the ED problem is 6. As shown in Table 2, there are 12 prohibited zones in this system, and 13 inequality constraints are generated according to these prohibited zones.

System 2: this system has 15 thermal units, the characteristics of which are given in Tables 4 and 5. The load demand of the system is $2630 \mathrm{MW}$. Due to space constraints, the loss coefficient matrix is not listed. This system is a medium-scale system, and its ED problem has 15 dimensions. As shown in Table 5, the power generating units 2, 5, 6, and 12 have 11 prohibited zones. Therefore, according to the inequality constraints described above regarding its ED problem, the ED problem of this system is relatively difficult to optimize compared with System 1.

System 3: the system contains 40 units in a large-scale hybrid power generation system named Tai power system. The load demand of the system is $8550 \mathrm{MW}$. Due to space constraints, unit parameters and loss factors are not listed. The dimension of the ED problem of this system is 40 . In the ED problem of this system, each power generation unit has no prohibited zone, so there are fewer unequal constraints, but this does not significantly reduce the difficulty of the problem. The large size and multiple fuel options attribute of this system make the ED problem to be one of the most difficult to solve among the three systems.

4.2. Parameters Setting. For each of these three systems, the maximum number of iterations to execute the objective 
Input: Input parameters: $\sigma_{1}$ and $\sigma_{0}$

Output: Fitness value of EDPs

Step 1: Initialize the current position of each particle, and set its personal best position to be its current position;

Step 2: Set $t=0$;

Step 3: While the termination condition is not met, perform the following steps;

Step 4: Set $t=t+1$ and compute the mean best position $C_{t}^{i}$;

Step 5: From $i=1$ executes the following steps;

Step 6: Evaluate the value of $X$, and update $G_{t}$ and $P_{t}^{i}$;

Step 7: Update position of each particle according to (2);

Step 8: set $i=i+1$, and return to Setp 5 until $i=M$;

Step 9: Return to Step 3;

Step 10: Return result

Algorithm 1: The pseudocode of SG-QPSO algorithm.

TABLE 1: 6 units of power generation capacity and coefficient.

\begin{tabular}{lccccc}
\hline Unit & $P_{j}^{\min }$ & $P_{j}^{\max }$ & $a_{j}$ & $b_{j}$ & $c_{j}$ \\
\hline 1 & 100 & 500 & 240 & 7.0 & 0.0070 \\
2 & 50 & 200 & 200 & 8.5 & 0.0095 \\
3 & 80 & 300 & 220 & 11.0 & 0.0090 \\
4 & 50 & 150 & 200 & 10.5 & 0.0090 \\
5 & 50 & 220 & 220 & 12.0 & 0.0080 \\
6 & 50 & 120 & 190 & 0.0075 \\
\hline
\end{tabular}

TABLE 2: Slope limit and prohibited zones of the generator set 6-unit system.

\begin{tabular}{lcccc}
\hline Unit & $P_{j}^{0}$ & $U R_{j}$ & $D R_{j}$ & Prohibited zones \\
\hline 1 & 440 & 80 & 120 & {$[210,240][350,380]$} \\
2 & 170 & 50 & 90 & {$[90,110][140,160]$} \\
3 & 200 & 65 & 100 & {$[150,170][210,240]$} \\
4 & 150 & 50 & 90 & {$[80,90][110,120]$} \\
5 & 190 & 50 & 90 & {$[90,110][140,150]$} \\
6 & 110 & 50 & 90 & {$[75,85][100,105]$} \\
\hline
\end{tabular}

TABLe 3: Loss factor $b$ of 6 -unit system.

\begin{tabular}{lcccccc}
\hline$B_{i j}$ & 1 & 2 & 3 & 4 & 5 \\
\hline 1 & 0.0017 & 0.0012 & 0.0007 & -0.0001 & -0.0005 & -0.0002 \\
2 & 0.0012 & 0.0014 & 0.0009 & 0.0001 & -0.0006 & -0.0001 \\
3 & 0.0007 & 0.0009 & 0.0031 & 0.001 & -0.0006 \\
4 & -0.0001 & 0.0001 & 0 & -0.0006 & -0.0006 \\
5 & -0.0005 & -0.0006 & -0.001 & -0.0008 & -0.0002 & -0.0002 \\
6 & -0.0002 & -0.0001 & -0.0006 & 0.0001 & -0.0002 \\
$B_{0 i}$ & -0.0004 & -0.0001 & 0.0007 & 0.056 & & -0.0007 \\
$B_{00}$ & & & & 0.000 & \\
\hline
\end{tabular}

function of each optimization algorithm is set to 20,000 . Simultaneously, two sets of experiments are performed on each system for each algorithm. One has a population size of $M=100$ and a maximum number of generation of $G_{\max }=200$, another population size of $M=20$ and $G_{\max }=1000$. On each system, each algorithm performed 100 independent experiments with a given maximum generation $G_{\max }$ and population size $M$. The penalty coefficient in the objective function is set to $K_{t}=100 \sqrt{t}$, where $t$ is the current number of generations.
The other experimental configuration settings are as follows: The size of the crossover probability $p_{c}=0.8$, and the size of the mutation probability $p_{m}=0.1$ in GA; the constant mutation factor used by the $\mathrm{DE}$ algorithm is $f_{m}=0.4$, and the size of the crossover rate is $\mathrm{CR}=0.8$; for PSO with inertia weight, the inertia weight decreases linearly from 0.9 to 0.4 during the search process, the acceleration coefficients $c_{1}=c_{2}=2.0$ and $V_{\max }=\left(\left(P_{j}^{\max }-P_{j}^{\min }\right) / 2\right)$; for SG-QPSO, during the search process, the variance of the Gaussian distribution $\sigma$ decreases linearly from 5 to 0.001 . 
TABLE 4: Slope limit and prohibited zones of the generator set 15-unit system.

\begin{tabular}{lccccc}
\hline Unit & $P_{j}^{\min }$ & $P_{j}^{\max }$ & $a_{j}$ & $b_{j}$ & $c_{j}$ \\
\hline 1 & 150 & 455 & 671 & 10.1 & 10.2 \\
2 & 150 & 455 & 574 & 8.8 & 0.000299 \\
3 & 20 & 130 & 374 & 8.8 & 0.000183 \\
4 & 20 & 130 & 374 & 10.4 & 0.001126 \\
5 & 150 & 470 & 461 & 9.8 & 0.001126 \\
6 & 135 & 460 & 630 & 11.2 & 0.000301 \\
& 135 & 465 & 548 & 11.2 & 0.000364 \\
8 & 60 & 300 & 227 & 10.7 & 0.000338 \\
9 & 25 & 162 & 173 & 10.2 & 0.000807 \\
10 & 25 & 160 & 175 & 9.9 & 0.001203 \\
11 & 20 & 80 & 186 & 0.003586 \\
12 & 20 & 80 & 230 & 13.1 & 0.005513 \\
13 & 25 & 85 & 225 & 12.1 & 0.000371 \\
14 & 15 & 55 & 309 & 0.001929 \\
15 & 15 & 55 & 323 & 0.004447 \\
\hline
\end{tabular}

TABLE 5: Slope limitation and prohibition zone for the 15-unit system of the generator set.

\begin{tabular}{lcccc}
\hline Unit & $P_{j}^{0}$ & $U R_{j}$ & $D R_{j}$ & Prohibited zones \\
\hline 1 & 400 & 80 & 120 & \\
2 & 300 & 80 & 120 & 130 \\
3 & 105 & 130 & 130 & \\
4 & 100 & 130 & $1225][305,335][420,450]$ \\
5 & 90 & 80 & 120 & {$[230,255][365,395][430,455]$} \\
6 & 400 & 80 & 120 & 120 \\
7 & 350 & 80 & 100 & 100 \\
8 & 95 & 65 & 100 & {$[30,40][55,65]$} \\
9 & 105 & 60 & 80 & 80 \\
10 & 110 & 60 & 80 & \\
11 & 60 & 80 & 55 & \\
12 & 40 & 80 & 55 & \\
13 & 30 & 80 & 55 & \\
14 & 20 & 55 & & \\
15 & 20 & $5390,420]$ \\
\hline
\end{tabular}

The algorithm parameters of ACSA, BCO, AIS, and FA are set according to the corresponding literature. The parameter configuration of other PSO variables, namely SPSO, CPSO, APSO, HGPSO, HPSOM, QPSO, and GQPSO, is the same as the parameters suggested in the literature. The parameter setting of Hopfield NN is consistent with that in literature.

4.3. Experiment Results and Analysis. Table 6 lists the total cost of each method for the ED problem of System 1. From Table 6, the average cost and standard deviation of 100 runs of SG-QPSO are better than other methods, which shows that the performance and robustness of SG-QPSO on System 1 is better than other algorithms. Under the two experimental configurations, the CPSO algorithm is the second best method of the system in terms of the mean cost. When $M=100$ and $G_{\max }=200$, the worst performance optimization algorithm is APSO, and the mean cost obtained in 100 runs is $15473.3164 \$ / h$. For this system, Hopfield NN produces the worst results. When $M=20$ and $G_{\max }=1000$, the mean cost of SPSO performs the worst, although the lowest cost found in 100 runs is $15442.9130 \$ / \mathrm{h}$, which is better than other comparison algorithms other than SG-QPSO. When $M=100$ and $G_{\max }=200$, the SG-QPSO algorithm obtained the best solution, the lowest standard deviation, and the best mean cost.

Table 7 lists the solution vector $P_{j}(j=1,2, \ldots, 6)$ relative to the best solution. The minimum cost of SG-QPSO running 100 times is $15442.7831 \$ / \mathrm{h}$ when $M=100$ and $G_{\max }=200$. To prove that the equality constraints in (16) are satisfied, we add the power loss $(12.4173 \mathrm{MW})$ to the load demand $(1263 \mathrm{MW})$ for a total of $1275.4173 \mathrm{MW}$. By comparing the sum to the total power output (1276.4183 MW), we can find that the equality constraints (i.e., power balance constraints) are well satisfied. Figure 1 visualizes the convergence of all test methods on the ED problem of System 1 for an average of 100 experiments, indicating that SG-QPSO has better convergence than other algorithms.

Table 8 lists the mean cost, minimum and maximum cost, and the standard deviation values obtained by performing 100 experiments with each algorithm of the ED 
TABLe 6: Results obtained by System 1 (6 unit system).

\begin{tabular}{|c|c|c|c|c|}
\hline & Min. Cost & Mean. Cost & Std. Cost & Max. Cost \\
\hline Hopfield NN & 15485.9374 & 15485.9374 & 0 & 15485.9374 \\
\hline \multicolumn{5}{|c|}{$M=100, G_{\max }=200$} \\
\hline GA & 15445.5961 & 15465.1757 & 9.7336 & 15491.4797 \\
\hline $\mathrm{DE}$ & 15444.9466 & 15450.1339 & 6.9854 & 15472.0651 \\
\hline ACSA & 15445.3052 & 15459.5170 & 12.0247 & 15511.5269 \\
\hline $\mathrm{BCO}$ & 15444.5837 & 15459.9441 & 8.4816 & 15482.3963 \\
\hline AIS & 15446.3283 & 15456.6660 & 7.3954 & 15481.2766 \\
\hline FA & 15445.9448 & 15461.3003 & 9.3385 & 15501.3958 \\
\hline PSO & 15444.7756 & 15466.5658 & 7.9185 & 15483.9700 \\
\hline SPSO & 15443.0188 & 15452.4764 & 9.5316 & 15490.2621 \\
\hline CPSO & 15442.9892 & 15449.1213 & 5.8048 & 15466.3953 \\
\hline APSO & 15445.5109 & 15473.3164 & 12.9048 & 15538.6016 \\
\hline HGPSO & 15447.1055 & 15462.6151 & 10.6456 & 15497.0335 \\
\hline HPSOM & 15443.6281 & 15449.2603 & 6.2745 & 15479.8640 \\
\hline QPSO & 15442.9803 & 15455.6220 & 11.6388 & 15482.2709 \\
\hline G-QPSO & 15443.2303 & 15473.3521 & 42.1254 & 15493.2412 \\
\hline SG-QPSO & 15442.7831 & 15445.0319 & 3.2756 & 15455.3582 \\
\hline \multicolumn{5}{|c|}{$M=20, G_{\max }=1000$} \\
\hline GA & 15446.4787 & 15465.9948 & 10.7090 & 15493.2033 \\
\hline $\mathrm{DE}$ & 15442.9836 & 15455.2537 & 13.7447 & 15489.8981 \\
\hline ACSA & 15445.3052 & 15462.5170 & 12.0247 & 15511.5269 \\
\hline $\mathrm{BCO}$ & 15446.3788 & 15461.8471 & 11.6661 & 15503.2901 \\
\hline AIS & 15443.1652 & 15458.0859 & 8.5894 & 15481.0627 \\
\hline FA & 15447.1955 & 15466.3277 & 12.5520 & 15512.0039 \\
\hline PSO & 15443.8360 & 15459.9688 & 13.3737 & 15529.6094 \\
\hline SPSO & 15442.9130 & 15477.9615 & 33.7155 & 15597.4534 \\
\hline CPSO & 15443.1280 & 15454.1791 & 8.0500 & 15460.4890 \\
\hline APSO & 15444.8934 & 15459.4084 & 11.0307 & 15493.3795 \\
\hline HGPSO & 15445.4041 & 15464.3992 & 11.5621 & 15494.0358 \\
\hline HPSOM & 15443.1716 & 15464.8451 & 22.8483 & 15533.1786 \\
\hline QPSO & 15443.0583 & 15465.4058 & 25.8660 & 15518.4093 \\
\hline G-QPSO & 15443.5143 & 15469.2825 & 56. 4224 & 1503.1225 \\
\hline SG-QPSO & 15442.8105 & 15453.9682 & 13.1657 & 15482.7553 \\
\hline
\end{tabular}

TABLE 7: $M=100, G_{\max }=200$ SG-QPSO system 1 (6-unit system).

\begin{tabular}{|c|c|c|c|c|}
\hline \multicolumn{5}{|c|}{ Power output: $P_{1}(\mathrm{MW}) \sim P_{6}(\mathrm{MW})$} \\
\hline $\begin{array}{ll}445.5381 & 172.8535\end{array}$ & 263.7547 & 141.3865 & 163.7148 & 89.1707 \\
\hline Total power output(MW) & & & 1276.4183 & \\
\hline Power loss(MW) & & & 12.4173 & \\
\hline Total generation $\operatorname{cost}(\$ / \mathrm{h})$ & & & 15442.7631 & \\
\hline
\end{tabular}

The best solution is obtained with 100 runs.

problem on System 2. Obviously, in any experiment configurations, SG-QPSO obtained the lowest cost. It can be seen that when $M=100$ and $G_{\max }=200$, QPSO ranks second with a mean cost of $15455.6220 \$ / \mathrm{h}$. When $M=20$ and $G_{\max }=1000$, the second-best method is HPSOM, and its average cost is $32811.3701 \$ / \mathrm{h}$. In the two experimental settings, the worst-performing optimization algorithms are BCO (mean cost 33113. 0149\$/h) and GA (mean cost $33188.5443 \$ / \mathrm{h})$

Table 9 lists the results of the ED problem of each algorithm on System 3. From this table, in all the algorithms of the two experiment configurations, SG-QPSO achieved the best results. When $M=100, G_{\max }=200$, the second-best performing algorithm is HPSOM, and the average cost obtained in 100 experiments is $131614.7211 \$ / \mathrm{h}$. In this set of experiments, GA performed the worst among all the algorithms participating in the test.

In two different experiments configurations, the best solution to the system is obtained through the SG-QPSO algorithm. The minimum cost of the algorithm in 100 runs was $32663.2635 \$ / h$. Table 10 shows the corresponding total power generation cost for the best solution is $32663.2635 \mathrm{\$} / \mathrm{h}$ when $M=20$ and $G_{\max }=1000$. From Table 9, the difference between the available load (2659.5748 MW-29.5683 MW) and the load demand (2630 MW) is 0.0065 , which proves that the power balance equation constraint in (9) and (18) is satisfied. Figure 2 shows that for this ED problem, the SGQPSO method has better convergence than other 


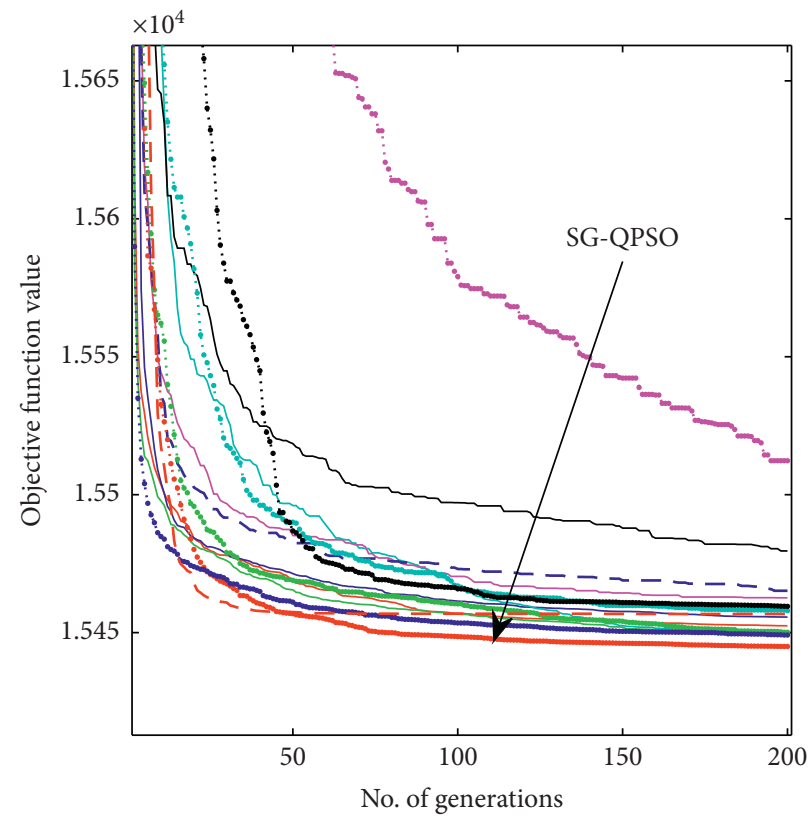

(a)

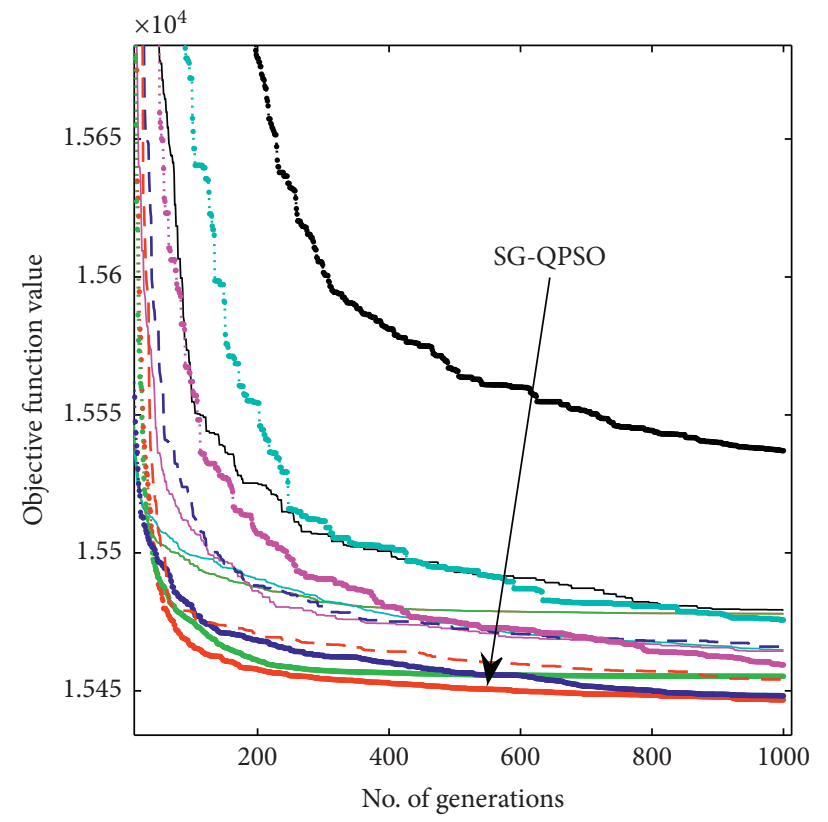

(b)

FIGURE 1: Convergence properties of the tested optimization methods for the 6-unit system with (a) $M=100$ and $G_{\max }=200$; (b) $M=20$ and $G_{\max }=1000$.

TABle 8: Results obtained by System 2 (15 unit system).

\begin{tabular}{|c|c|c|c|c|}
\hline & Min. cost & Mean. cost & Std. cost & Max. cost \\
\hline Hopfield NN & 34281.4857 & 34281.4857 & 0 & 34281.4857 \\
\hline \multicolumn{5}{|c|}{$M=100, G_{\max }=200$} \\
\hline GA & 32939.5208 & 33106.0019 & 100.1279 & 33231.6216 \\
\hline $\mathrm{DE}$ & 32818.5792 & 32990.8673 & & 33116.9340 \\
\hline ACSA & 32785.6031 & 33051.7711 & 77.80 & 33185.2761 \\
\hline $\mathrm{BCO}$ & & & & 4940 \\
\hline AIS & 2895.9173 & & & 0191 \\
\hline FA & 32901.6610 & 3308 & 91.0 & 2718 \\
\hline PSO & 32715.0 & 3294 & 121.8 & 0099 \\
\hline & & & & 619 \\
\hline CPS & & & & 0568 \\
\hline APS & & & & 5609 \\
\hline & & & & 2655 \\
\hline & & & & 284 \\
\hline & & & & 0659 \\
\hline G-Q & 02.2125 & & & 1642 \\
\hline & 771.2583 & 32745.5195 & & \\
\hline \multicolumn{5}{|c|}{$M=20, G_{\max }=1000$} \\
\hline GA & 905.3592 & 33188.5443 & & \\
\hline $\mathrm{DE}$ & 32718.8201 & 32966.4332 & 110.32378 & 33213.3173 \\
\hline ACSA & 32863.1770 & 33120.0202 & & 33256.2899 \\
\hline $\mathrm{BCO}$ & & & & 33301.4940 \\
\hline AIS & & & & .0191 \\
\hline FA & 32898.0118 & 33116.9006 & 96.3875 & 33310.7299 \\
\hline PSO & 32735.6944 & 33039.0837 & 102.0513 & 33297.2240 \\
\hline SPSO & & & & 33399.6968 \\
\hline CPSO & & & 112. & 2.1291 \\
\hline APSO & 32861.4413 & 32996.4562 & 84.8760 & 33255.0095 \\
\hline & 32782.2876 & 33019.8081 & 139.8065 & 33413.7438 \\
\hline & 32677.3925 & 32811.3701 & & 32992.3424 \\
\hline & & & & 33106.1779 \\
\hline & 36932.1185 & 32833.3232 & 183.1024 & 33132.4262 \\
\hline SG-QPSO & 32663.2635 & 32758.6330 & 84.1633 & 32973.1083 \\
\hline
\end{tabular}

TABle 9: Results obtained by System 3 (40-unit system).

\begin{tabular}{|c|c|c|c|c|}
\hline & Min. Cost & Mean. Cost & Std. Cost & Max. Cost \\
\hline $\begin{array}{l}\text { Hopfield } \\
\text { NN }\end{array}$ & 136443.7361 & 136443.7361 & 0 & \\
\hline \multicolumn{5}{|c|}{$M=100, G_{\max }=200$} \\
\hline GA & 133032.8559 & 135162.1174 & 766.2829 & 137015.1418 \\
\hline $\mathrm{DE}$ & 130560.8784 & 1317 & 672.3 & 1490 \\
\hline & & & & .9684 \\
\hline $\mathrm{BCO}$ & & 132 & & .5434 \\
\hline AIS & 065 & 1326 & & 6066 \\
\hline & 80 & 133 & & 4171 \\
\hline & & & & \\
\hline & & & & 8482 \\
\hline & & & & \\
\hline & & & & \\
\hline & & & & 1546 \\
\hline & & & & \\
\hline & 48 & & & 7305 \\
\hline & & & & 2331 \\
\hline & 7947 & 131269.5009 & & \\
\hline \multicolumn{5}{|c|}{$M=20, G_{\max }=1000$} \\
\hline GA & & 135012.4985 & & \\
\hline $\mathrm{DE}$ & & & & 9461 \\
\hline & & & & \\
\hline $\mathrm{BCO}$ & & & & \\
\hline AIS & .9214 & 13148 & 561.79 & 3.1884 \\
\hline FA & & & & 134997.9243 \\
\hline & 7.0844 & 132614.1979 & & 135008.7394 \\
\hline & & & 137 & 138444.9147 \\
\hline CPSO & 129638.4548 & 130812.0434 & 651.0647 & 134184.2693 \\
\hline & 130861.5242 & 132587.8486 & 675.0306 & 134044.6303 \\
\hline & 132072.2495 & 134012.5706 & 684.4951 & 135528.3862 \\
\hline & & & & \\
\hline & 129519.5044 & 130498.1964 & 573.5890 & 132264.9375 \\
\hline & 131213.3145 & 131417.2211 & 598.2213 & 133102.1132 \\
\hline SG-QPSO & 129078.4705 & 129884.6948 & 549.4955 & 131198.4069 \\
\hline
\end{tabular}


TABLe $10: M=20, G_{\max }=1000$ SG-QPSO system 2 (15-unit system).

\begin{tabular}{lcccc}
\hline & & & \\
\hline$P_{1} \sim P_{4}(\mathrm{MW})$ & 455.1085 & 380.9460 & 126.6947 & 127.8492 \\
$P_{5} \sim P_{8}(\mathrm{MW})$ & 170.7069 & 463.8439 & 427.6885 & 75.3608 \\
$P_{9} \sim P_{12}(\mathrm{MW})$ & 50.7581 & 163.3610 & 77.6944 & 80.5684 \\
$P_{13} \sim P_{15}(\mathrm{MW})$ & 25.7335 & 20.5853 & 12.6756 & \\
\hline \multicolumn{2}{c}{ Total power output(MW) } & 2659.5748 & Power loss $(\mathrm{MW})$ & 32663.2635 \\
\hline \multicolumn{2}{c}{ Total generation $\operatorname{cost}(\$ / \mathrm{h})$} & & & 29.5683 \\
\hline
\end{tabular}

The best solution is obtained with 100 runs.

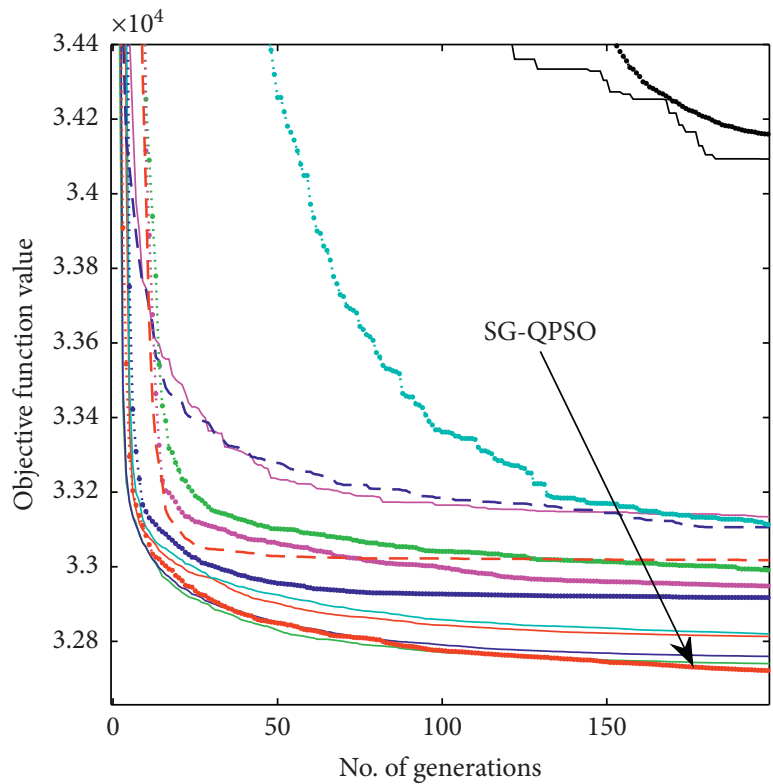

(a)

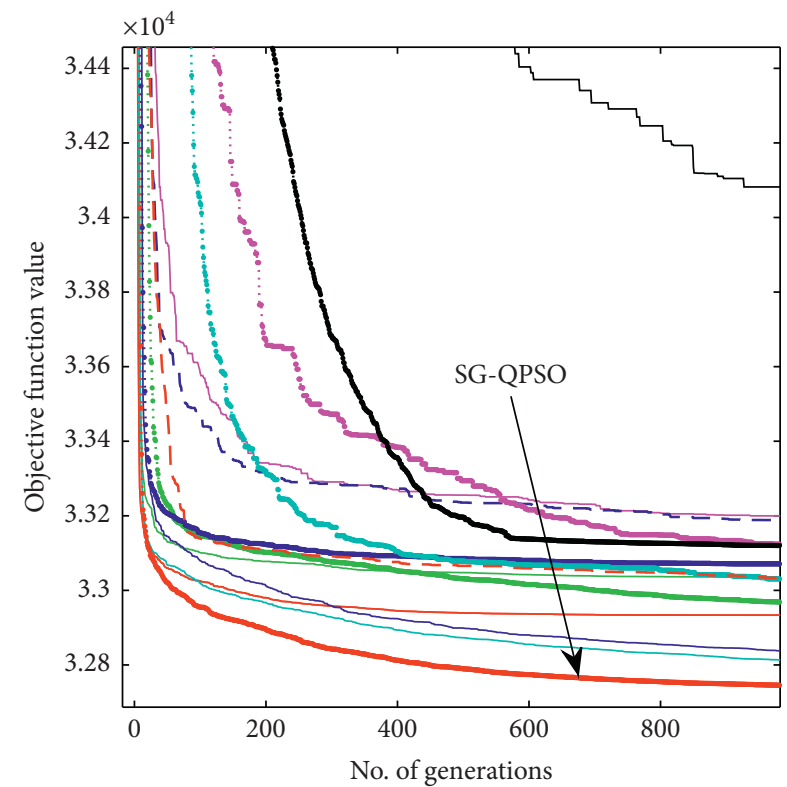

(b)

Figure 2: Convergence properties of the tested optimization methods for the 15 -unit system with (a) $M=100, G_{\max }=200$; (b) $M=20, G_{\max }=1000$.

TABLE 11: The best solution obtained by using SG-QPSO for System 3 (40-unit system).

Total Power Output(MW) 8631.9425

Power loss(MW)

81.9390

Total generation $\operatorname{cost}(\$ / \mathrm{h})$

129078.4705

Use $M=20, G_{\max }=1000$ (100 runs).

algorithms, providing faster convergence speed and the best final mean fitness value.

From Table 9, when $M=20, G_{\max }=1000$, the SG-QPSO algorithm can obtain the best solution to the ED problem of the system. Due to the space limitations of this article, we only list the best results of total power output and system transmission loss obtained when the minimum total cost is $129078.4705 \$ / h$ in Table 11 . In order to prove that the equality constraints in (9) and (18) are satisfied, we will combine the left side of (9) (that is, total output power (8631.9425 MW)) and the right side of (9) (that is, power loss (81.9390 MW)) and the sum of the load demand (8550 MW) (8631.9425 MW) is compared, and both satisfy the equality constraints. In addition, as shown in Figure 3, SG-QPSO also has the best convergence for the fitness value of the ED problem of the system. 


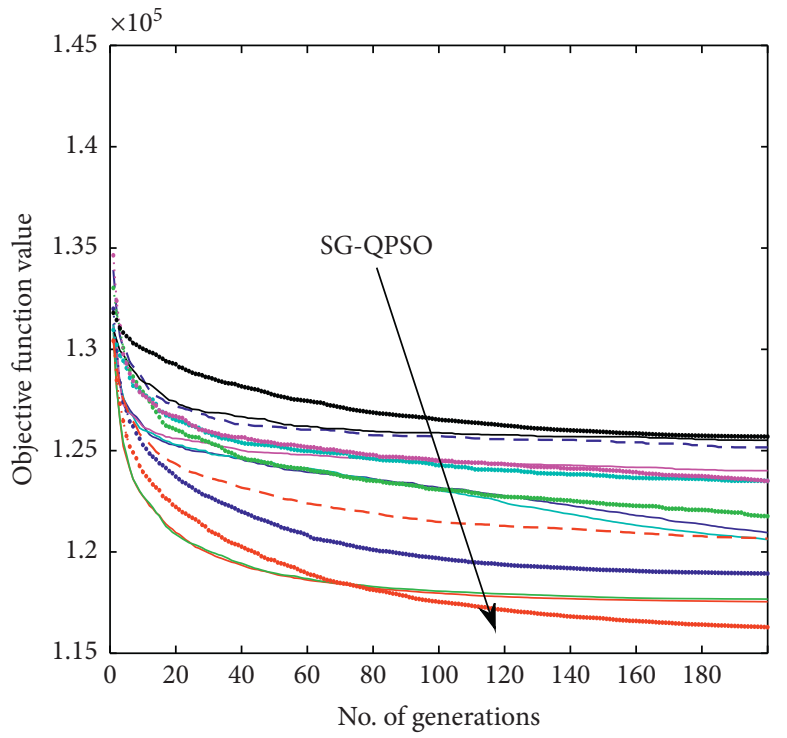

(a)

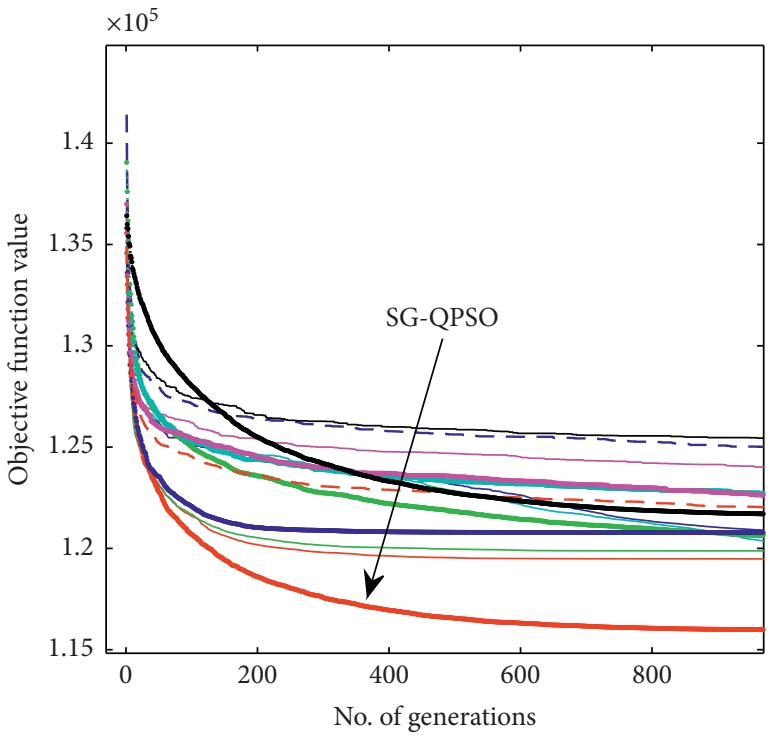

(b)

Figure 3: Convergence properties of the tested optimization methods for the 40 -unit system with (a) $M=100, G_{\max }=200$; (b) $M=20, G_{\max }=1000$.

\section{Conclusion}

A shrink Gaussian distribution Quantum-behaved particle swarm optimization (SG-QPSO) algorithm is proposed to effectively solve the power economic dispatch problem by considering the nonlinear characteristics of the generator. SG-QPSO yields better solutions of different systems compared to any other tested algorithms, and highly similar optimization results among 100 independent trails of each system confirmed its robustness. In addition, the performance of SG-QPSO shows a stronger global search performance, which can be seen from the relatively low system cost obtained in 100 runs. Therefore, the SG-QPSO method is a promising tool for solving ED problems and other optimization problems in the industrial field. Our future work will focus on the application of the SG-QPSO method on other industrial problems, as well as the theoretical analysis of the algorithm search mechanism.

\section{Data Availability}

The experimental data used to support the findings of this study are included within the article.

\section{Conflicts of Interest}

The authors declare that they have no conflicts of interest.

\section{Acknowledgments}

This work was partially supported by the National Natural Science Foundation of China (61672263) and sponsored by Qing Lan Project.

\section{References}

[1] B. H. Chowdhury and S. Rahman, "A review of recent advances in economic dispatch," IEEE Transactions on Power Systems, vol. 5, no. 4, pp. 1248-1259, 1990.

[2] S. Granville, "Optimal reactive dispatch through interior point methods," IEEE Transactions on Power Systems, vol. 9, no. 1, pp. 136-146, 1994.

[3] R. N. Dhar and P. K. Mukherjee, "Reduced-gradient method for economic dispatch," Proceedings of the Institution of Electrical Engineers, vol. 120, no. 5, pp. 608-610, 1973.

[4] Z.-X. Liang and J. D. Glover, "A zoom feature for a dynamic programming solution to economic dispatch including transmission losses," IEEE Transactions on Power Systems, vol. 7, no. 2, pp. 544-550, 1992.

[5] P.-H. Chen and H.-C. Chang, "Large-scale economic dispatch by genetic algorithm," IEEE Transactions on Power Systems, vol. 10, no. 4, pp. 1919-1926, 1995.

[6] Z.-L. Gaing, "Particle swarm optimization to solving the economic dispatch considering the generator constraints," IEEE Transactions on Power Systems, vol. 18, no. 3, pp. 1187-1195, 2003.

[7] L. S. Coelho and V. C. Mariani, "Combing of chaotic differential evolution and quadratic programming for economic dispatch optimization with valve-point effect," IEEE Transactions on Power Systems, vol. 21, no. 2, pp. 989-996, 2006.

[8] Y. M. Park, J. R. Won, and J. B. Park, "A new approach to economic load dispatch based on improved evolutionary programming," Engineering Intelligent Systems for Electrical Engineering and Communications, vol. 6, no. 2, pp. 103-110, 1998.

[9] N. Sinha, R. Chakrabarti, and P. K. Chattopadhyay, "Evolutionary programming techniques for economic load dispatch," IEEE Transactions on Evolutionary Computation, vol. 7, no. 1, pp. 83-94, 2003.

[10] W. M. Lin, F. S. Cheng, and M. T. Tsay, “An improved Tabu search for economic dispatch with multiple minima," IEEE Transactions on Magnetics, vol. 38, pp. 1037-1040, 2002. 
[11] C. Yang, G. Deconinck, and W. Gui, "An optimal powerdispatching control system for the electrochemical process of zinc based on backpropagation and hopfield neural networks," IEEE Transactions on Industrial Electronics, vol. 50, no. 5, pp. 953-961, 2003.

[12] K. Y. Lee, A. Sode-Yome, and J. H. June Ho Park, "Adaptive hopfield neural networks for economic load dispatch," IEEE Transactions on Power Systems, vol. 13, no. 2, pp. 519-526, 1998.

[13] T. Sum-Im, "Economic dispatch by ant colony search algorithm," in Proceedings of the 2004 IEEE Conference Cyber Intelligence Systems, pp. 416-421, Singapore, December 2004.

[14] T. K. Abdul Rahman, Z. M. Yasin, and W. N. W. Abdullah, "Artificial-immune-based for solving economic dispatch in power system," in Proceedings of the 2004 National Power Energy Conference, pp. 31-35, Kuala Lumpur, Malaysia, November 2004.

[15] Y. Chuan, G. K. Venayagamoorthy, and K. Corzine, "AISbased coordinated and adaptive control of generator excitation systems for an electric ship," IEEE Transactions on Industrial Electronics, vol. 59, no. 8, pp. 3102-2112, 2012.

[16] C. Chokpanyasuwan, "Honey bee colony optimization to solve economic dispatch problem with generator constraints," in Proceedings of the 6th International Conference on Electrical Engineering/Electronics, Computer, Telecommunications and Information Technology, pp. 200-203, Pattaya, Thailand, May 2009.

[17] X.-S. Yang, S. S. Sadat Hosseini, and A. H. Gandomi, "Firefly algorithm for solving nonconvex economic dispatch problems with valve loading effect," Applied Soft Computing, vol. 12, no. 3, pp. 1180-1186, 2012.

[18] C.-P. Cheng, C.-W. Liu, and C.-C. Liu, "Unit commitment by annealing-genetic algorithm," International Journal of Electrical Power \& Energy Systems, vol. 24, no. 2, pp. 149-158, 2002.

[19] S. H. A. Kaboli and A. K. Alqallaf, "Solving nonconvex economic load dispatch problem via artificial cooperative search algorithm," Expert Systems with Applications, vol. 128, pp. 14-27, 2019.

[20] V. C. Pandey, V. K. Jadoun, N. Gupta, K. R. Niazi, and A. Swarnkar, "Improved fireworks algorithm with chaotic sequence operator for large-scale nonconvex economic load dispatch problem," Arabian Journal for Science and Engineering, vol. 43, no. 6, pp. 2919-2929, 2018.

[21] J. Sun, V. Palade, X.-J. Wu, W. Fang, and Z. Wang, "Solving the power economic dispatch problem with generator constraints by random Drift particle swarm optimization," IEEE Transactions on Industrial Informatics, vol. 10, no. 1, pp. 222-232, 2014.

[22] S. Khamsawand, P. Wannakarn, and S. Jiriwibhakorn, "Hybrid PSO-DE for solving the economic dispatch problem with generator constraints," in Proceedings of the 2nd International Conference on Computer and Automation Engineering, pp. 135-139, IEEE, Singapore, Feburary 2010.

[23] H. Garg, "A hybrid PSO-GA algorithm for constrained optimization problems," Applied Mathematics and Computation, vol. 305, pp. 274-292, 2016.

[24] S. Khan, O. U. Rehman, N. Khan et al., "Improving the diversity of PSO for an engineering inverse problem using adaptive inertia weight," Tehnicki Vjesnik Technical Gazette, vol. 25 , no. $6,2018$.

[25] E. Comak, "A particle swarm optimizer with modified velocity update and adaptive diversity regulation," Expert Systems, vol. 36, no. 1, pp. 1-18, Article ID e12330, 2019.
[26] Z. Liu, H. Li, and P. Zhu, "Diversity enhanced particle swarm optimization algorithm and its application in vehicle lightweight design," Journal of Mechanical Science and Technology, vol. 33, no. 2, pp. 695-709, 2019.

[27] M. Modiri, S. H. Aghaykaboli, E. Taslimi et al., "An iteratedbased optimization method for economic dispatch in power system," in Proceedings of the 2013 IEEE Conference on Clean Energy and Technology (CEAT), IEEE, Lankgkawi, Malaysia, November 2014.

[28] V. K. Jadoun, N. Gupta, K. R. Niazi, and A. Swarnkar, "Dynamically controlled particle swarm optimization for large-scale nonconvex economic dispatch problems," International Transactions on Electrical Energy Systems, vol. 25, no. 11, pp. 3060-3074, 2015.

[29] J. Sun, W. Xu, and B. Feng, "A global search strategy of quantum-behaved particle swarm optimization," in Proceedings of the 2004 IEEE Conference on Cybernetics and Intelligent Systems, pp. 111-116, IEEE, Singapore, December 2004.

[30] M. Clerc, "Stagnation analysis in particle swarm optimization or what happens when nothing happens [EB/OL]," 2019, http://clerc.maurice.free.fr/pso/.

[31] S. H. Ling, H. H. C. Iu, K. Y. Chan, H. K. Lam, B. C. W. Yeung, and F. H. Leung, "Hybrid particle swarm optimization with wavelet mutation and its industrial applications," IEEE Transactions on Systems, Man, and Cybernetics, Part B (Cybernetics), vol. 38, no. 3, pp. 743-763, 2008.

[32] J. Cai, X. Ma, L. Li, and P. Haipeng, "Chaotic particle swarm optimization for economic dispatch considering the generator constraints," Energy Conversion and Management, vol. 48, no. 2, pp. 645-653, 2007.

[33] A. I. Selvakumar and K. Thanushkodi, "Anti-predatory particle swarm optimization: solution to nonconvex economic dispatch problems," Electric Power Systems Research, vol. 78, pp. 2-10, 2008.

[34] L. d. S. Coelho, "Gaussian quantum-behaved particle swarm optimization approaches for constrained engineering design problems," Expert Systems with Applications, vol. 37, no. 2, pp. 1676-1683, 2010. 